\title{
Enjeux, méthodes et fondamentaux de prévisibilité et prévision décennale
}

\author{
Christophe Cassou ${ }^{(1)}$ et Juliette Mignot ${ }^{(2)}$ \\ (1) CNRS-CERFACS \\ (2) IRD-IPSL LOCEAN et KUP-Univ Berne
}

\section{Résumé}

Dans le cadre du $5^{\mathrm{e}}$ exercice international d'intercomparaison des modèles couplés, la communauté scientifique a conduit, pour la première fois à l'échelle du Programme mondial de recherche sur le climat, un ensemble coordonné de simulations dit de prévision décennale. Le but de cette initiative est de mieux comprendre les modulations climatiques qui s'expliquent en grande partie à l'échelle de temps de 5-20 ans par la variabilité interne de l'océan et qui se superposent aux tendances à long terme, telles que le réchauffement d'origine anthropique. Il s'agit ensuite d'estimer la prévisibilité de ces modulations, en particulier aux échelles régionales. Les prévisions décennales diffèrent des projections climatiques traditionnelles par l'étape d'initialisation des modèles aux observations directes ou à leurs estimations via les réanalyses. Leur vocation est de reproduire les fluctuations observées, contrairement aux projections climatiques et simulations historiques, qui ne visent qu'à estimer la réponse du système climatique aux forçages dits externes, tels l'activité solaire, le volcanisme et les perturbations anthropiques. Le but de cet article est de présenter les fondamentaux de la prévision décennale, du concept aux méthodes en passant par les difficultés et les limites, puis de donner quelques premiers résultats de prévisibilité à partir de prévisions rétrospectives sur la période 19602010. a prévision décennale est une des principales nouveautés du $5^{\mathrm{e}}$ rapport du GIEC $^{(1)}$ dont la publication du premier volet est prévue pour fin 2013. On trouve son empreinte dans tous les grands projets internationaux sous la houlette du Programme mondial de recherche sur le climat (PMRC), notamment l'exercice d'intercomparaison de modèles climatiques (CMIP5) ${ }^{(2)}$ qui en fournit le cadre ou encore dans les divers appels d'offre de la Commission européenne. La prévision décennale se niche aussi dans les priorités et prospectives de recherche des organismes et agences nationales, tels l'Institut national des sciences de l'univers (INSU) sous la tutelle du $\mathrm{CNRS}^{(3)}$, l'Agence nationale de la recherche (ANR), les programmes animés par le ministère de l'Écologie, du Développement durable et de l'Energie comme le $\mathrm{GICC}^{(4)}$, etc.

L'engouement récent pour la prévision décennale s'explique par trois facteurs. Le premier repose sur le constat que le réchauffement global des années 2000 est plus lent que celui des années 1980 et 1990, lequel était plus rapide que celui des décennies précédentes, même si 8 années de la décennie 2000-2009 se classent dans le « top 10 » des années les plus chaudes observées depuis le début de l'ère instrumentale, c'est-à-dire depuis $1880^{(5)}$. La question est donc, d'une part, de déterminer quels sont les mécanismes physiques à l'origine de cette modulation aux échelles de la décennie et, d'autre part, d'évaluer son degré de prévisibilité, afin de réduire les incertitudes (Hawkins et Sutton, 2009) sur les changements à venir dans un futur proche (2010-2035).
Le deuxième facteur découle des progrès du duo modélisation/observation qui autorisent désormais à envisager la prévision climatique à ces échelles de temps longues. La prévision décennale est en effet à double contrainte, car elle repose sur l'utilisation de modèles climatiques incluant les principales composantes du système (atmosphère, océan, cryosphère, surfaces continentales), leurs interactions et leurs variations, et sur leur démarrage à partir des conditions observées. Les avancées en la matière sont importantes, avec, d'un coté, l'émergence de modèles de plus en plus complexes capables de reproduire, de manière satisfaisante, les grandes fluctuations climatiques du passé (Braconnot et al., 2012 ; Swingedouw et al., 2011) et, de l'autre, l'émergence de nouveaux systèmes d'observations, en particulier les flotteurs Argo qui assurent une couverture tridimensionnelle de l'océan (de la surface jusqu'à $2000 \mathrm{~m}$ de fond en température, salinité et courant), les produits satellitaires permettant de quantifier avec précision les échanges entre sous-systèmes climatiques (GRACE pour le bilan d'eau, le programme CERES pour les flux d'énergie à la surface).

Le troisième facteur repose sur l'adéquation entre l'échelle de temps décennale et l'échelle de temps des décisions et actions à prendre par la sphère sociétale, économique et politique dans un contexte d'adaptation au changement climatique. L'échelle décennale est davantage «palpable » pour les décideurs et le citoyen, ce qui est bien moins le cas des projections climatiques pour la fin $\mathrm{du} \mathrm{XXI}^{\mathrm{e}}$ siècle. Ces dernières 
furent au cœur du $4^{\mathrm{e}}$ rapport du GIEC de 2007 et furent largement relayées auprès du public via les académies, médias et organisations diverses.

\section{Abstract}

Challenges, basic principles and methodologies for decadal predictability and forecasts

Within the framework of the $5^{\text {th }}$ coupled model intercomparaison project, an ensemble of coordinated decadal forecast simulations has been carried out, for the first time, by the climate community. This new initiative aims at better understanding the decadal modulations, superimposed on the anthropogenic-forced warming tendency, and that are mostly explained by the internal variability in the ocean. The goal of this initiative is to provide a first assessment of the climate predictability at decadal timescale. Forecasts differ from climate projections by the mandatory step of initialization of the model components either to direct observations or to their reconstruction via the reanalyzes. They aim at simulating the observed fluctuations, whereas projections and historical simulations only estimate the climate response to so-called external forcings such as solar activity, volcanism and anthropogenic perturbations. The goal of this article is to review the basics of the decadal forecasts (concepts, methodologies, difficulties and limits) and to present preliminary and simple results on predictive skill estimated from hindcasts over 1960-2010.
A priori, présenté ainsi : " vous savez faire », dit-on à la communauté des climatologues. Pour certains non experts, il s'agit simplement d'intégrer les modèles de prévision saisonnière sur 10 ou 20 ans au lieu du standard 3-6 mois fonctionnant actuellement en production opérationnelle dans les grands centres météorologiques. Pour d'autres, il s'agit simplement de détailler sur 20102035 les scénarios et projections climatiques comme il est pratiqué couramment pour les études d'impact sur la seconde moitié du XXI ${ }^{e}$ siècle, quand le forçage anthropique domine. Ces deux fausses intuitions reposent sur une confusion entre «prévisions » et « projections » climatiques et sur une méconnaissance des hypothèses et fondamentaux de la modélisation climatique en mode prévision (Déqué, 2003) ainsi que de la nature et fiabilité des observations nécessaires. En prévision décennale, les protocoles expérimentaux et les hypothèses de travail classiques ne s'appliquent pas ou trouvent souvent leurs limites, car les questions d'interaction entre les différentes sources de variabilité climatique (et donc de prévisibilité) et les différentes sources d'incertitude, deviennent centrales.

Le but de cet article est de présenter les bases de la prévision décennale, c'est-àdire la nature physique et statistique du signal à prévoir à ces échelles de temps, les outils et méthodologies mis en œuvre, leurs limites, avant de conclure par les premiers résultats de cette prévisibilité.

Figure 1 - Histogramme des anomalies annuelles des températures de surface océanique (données ERSSTv3) moyennées sur l'Atlantique entre $0^{\circ}$ et $60^{\circ} \mathrm{N}$, calculées sur la période 1900-2010. Courbe magenta : évolution observée de la concentration du $\mathrm{CO}_{2}$ dans l'atmosphère. Courbe noire : comme l'histogramme mais après soustraction de la contribution du $\mathrm{CO}_{2}$ estimée par régression linéaire sur l'indice (trait plein) ou soustraction de la température océanique globale (trait pointillé), selon Trenberth et Shea (2006).

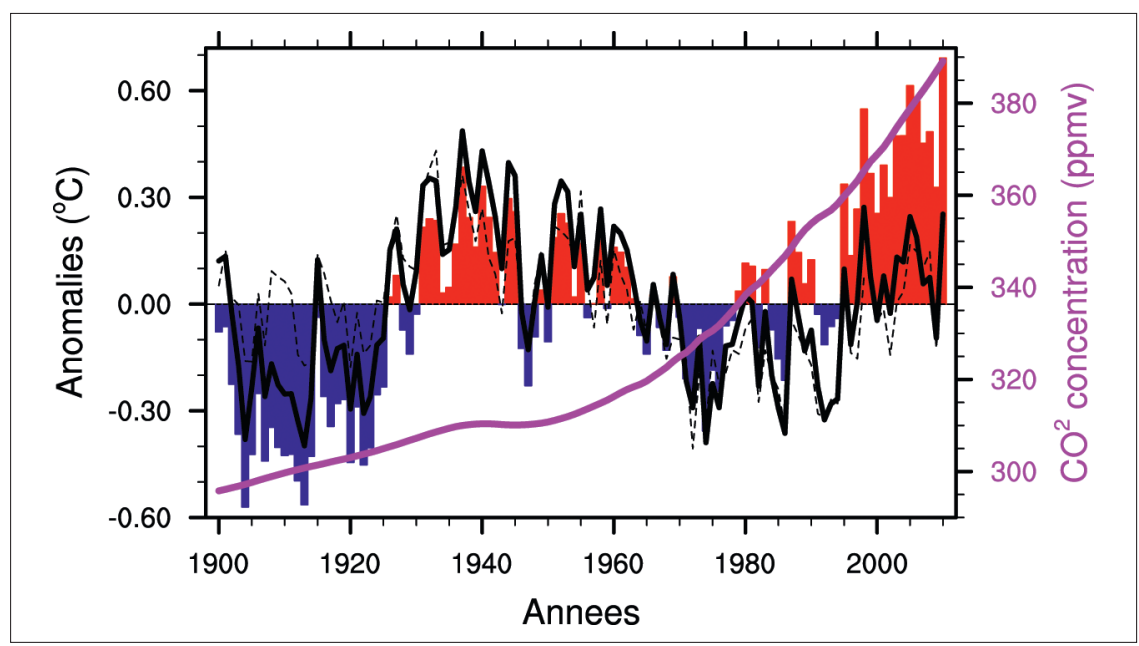

\section{La composante décennale d'un signal climatique : nature physique et prévisibilité}

Définissons tout d'abord le « système climatique ». Il s'agit du milieu dans lequel nous vivons, notre « environnement », l'enveloppe superficielle de la Terre regroupant des composantes très différentes telles que l'atmosphère, les océans, les surfaces continentales, etc. ainsi que toutes leurs interactions, physiques, chimiques et biologiques. L'évolution temporelle d'un signal (mesure locale ou intégrée) climatique peut se comprendre, en première approximation, comme le résultat de la superposition de deux sources de variabilité : l'une dite variabilité forcée induite par les changements des conditions aux limites du système climatique défini ci-dessus ou forçages externes, l'autre dite intrinsèque ou variabilité interne pilotée par les échanges d'énergie et d'eau entre les composantes climatiques de caractéristiques physiques très différentes (forte capacité thermique et circulations lentes pour les océans par opposition à l'atmosphère, rétention des écoulements d'eau dans la cryosphère - calotte, glaciers -, et les surfaces continentales, etc.). Les forçages externes sont de deux catégories : les forçages naturels (modulations de l'activité solaire, volcanisme) et les forçages d'origine anthropique correspondant aux émissions de gaz à effet de serre (GES), aux émissions d'aérosols et aux changements des conditions des sols (déforestation, agriculture, etc.) associés à l'activité humaine. Solaire, volcanisme et GES sont des forçages planétaires, alors que les aérosols et les sols s'expriment à des échelles plus régionales ${ }^{(1)}$. La prévision aux échelles de temps décennales consiste donc à prévoir, dans cette fenêtre temporelle, à la fois la réponse du système climatique à tous les forçages externes et l'évolution des modes de variabilité interne qui se superposent à cette réponse.

Prenons l'exemple du bassin atlantique nord. La figure 1 montre l'évolution des températures de surface océanique (ou SST, pour Sea Surface Temperature) moyennées entre $0^{\circ}$ et $60^{\circ} \mathrm{N}$ et présentées ici sous forme d'anomalies, c'està-dire d'écart à la valeur moyenne sur la période 1900-2010. L'évolution

(1) L'échelle régionale dans tout cet article correspond à l'échelle du continent ou du bassin océanique. 
temporelle de cet indice brut peut se décomposer en deux termes : une tendance au réchauffement particulièrement marquée depuis le milieu des années 1990 et un signal quasi oscillant, de période $~ 80$ ans, avec des années plus chaudes que la moyenne ( 1925-1965 et depuis 1995) et des décennies plus froides $(\sim 1900-1925$ et $\sim 1965-1995)$. Le premier terme est attribué majoritairement à la réponse de l'océan Atlantique au forçage par les GES d'origine anthropique (courbe magenta ; Terray, 2012). Le deuxième est appelé oscillation atlantique multidécennale (ci-après AMO ; Kerr, 2000) considéré pour une grande part comme un mode interne de variabilité du bassin atlantique. La manière classique de l'isoler (courbe noire) consiste à soustraire au signal total la contribution des GES par simple régression linéaire. Une autre manière est de soustraire au signal brut la SST moyennée sur l'ensemble de la planète (Trenberth et Shea, 2006). Cette moyenne peut en effet s'interpréter alors comme la meilleure estimation de la réponse du système climatique à tous les forçages externes planétaires (solaire, volcan et GES) confondus. Les deux techniques d'extraction de l'AMO donnent des résultats très comparables (courbe noire pointillée) confirmant le caractère dominant des GES dans les tendances globales récentes, en particulier depuis les années 1970.

On réalise tout de suite que, pour prévoir par exemple le fort réchauffement de la décennie 1990-2000 sur l'Atlantique nord (figure 1), il faut correctement estimer à la fois la tendance au réchauffement global et la bascule en phase chaude de l'AMO autour de 1995, les deux signaux étant d'amplitude comparable. Le succès de la prévision ne peut reposer que sur la prise en compte de ces deux sources de prévisibilité. Associée à la tendance, la première source (dite de « prévisibilité de deuxième espèce " selon la formulation de Lorenz, 1975) correspond à la prise en compte de l'évolution des forçages externes du système climatique. Associée à la bascule de l'AMO, la seconde (dite de «prévisibilité de première espèce $»)$, correspond à la connaissance des précurseurs physiques (intrinsèques au système climatique) à l'origine du changement de phase de l'AMO au milieu de la décennie 1990. Dans le jargon de la prévision, on parle de prévisibilité associée respectivement aux conditions aux limites et aux conditions initiales qui reposent beaucoup à l'échelle décennale sur la disponibilité et la qualité des observations océaniques. En effet, l'empreinte de l'AMO ne se limite pas à l'océan de surface, mais correspond à une fluctuation de l'ensemble du contenu thermique stocké dans le réservoir océanique de l'Atlantique nord, mettant très probablement en jeu des modifications de la circulation profonde de type thermohaline (Knight et al., 2005).

L'exemple de l'AMO n'est pas anodin, l'enjeu lié à sa prévision est grand, car elle a une signature marquée en termes d'impacts : son empreinte est clairement détectable dans les fluctuations décennales de l'activité cyclonique dans l'arc caribéen au cours des 60 dernières années (Goldenberg et al., 2001) et dans les précipitations sahéliennes (Zhang et Delworth, 1996). Les grandes sécheresses des années 1970-1980 à l'origine de famines catastrophiques correspondent ainsi aux phases "froides " de l'Atlantique nord, alors que le retour à des moussons en moyenne plus nourries sur la décennie 2000 se cale sur la bascule de l'AMO en phase chaude au milieu des années 1990. En Europe, une phase chaude de l'AMO semble être aussi associée à des précipitations estivales plus marquées sur la façade océanique du continent, alors qu'elles sont déficitaires sur l'arc méditerranéen, et à des températures plus chaudes (Sutton et Dong, 2012). Dans le Pacifique, il existe aussi un mode interne de variabilité à fort impact aux échelles décennales : la PDO, pour Pacific Decadal Oscillation, avec une signature en SST essentiellement dans le bassin nord et tropical (Mantua et al., 1997). La PDO contrôle une grande fraction de variance basse fréquence des précipitations, et donc des ressources en eau sur le continent nord-américain, ou bien affecte fortement les écosystèmes (e.g. conditions favorables à la prolifération de certains parasites par la modification d'occurrence de vagues de froid, longues périodes de sécheresse). La prévision potentielle au sens climatique du terme (information probabiliste) de ces deux modes serait donc particulièrement utile.

\section{Outils et méthodes pour la prévision décennale}

L'outil du prévisionniste, quelles que soient les échelles de temps en question, est le modèle de circulation générale (ciaprès GCM) des enveloppes fluides, véritable maquette de l'enveloppe superficielle de la Terre. Les GCM simulent, d'une part, les mouvements planétaires de l'atmosphère, de l'océan et de la glace de mer selon les lois physiques de la mécanique des fluides sur une sphère en rotation et, d'autre part, les échanges thermodynamiques et de matière entre les sous-systèmes climatiques incluant les surfaces continentales (Déqué, 2003). Sont ainsi considérées les diverses sources d'énergie (radiation, dégagement de chaleur latente associée aux changements de phase de l'eau, etc.) et les diverses sources hydrologiques aux échelles planétaires (calotte, ruissellement de l'eau via les fleuves, etc.). Les GCM, plus ou moins raffinés selon l'échelle de temps considérée, sont confrontés de manière permanente aux observations dans le cadre des prévisions opérationnelles météorologique et climatique (échelle mensuelle à saisonnière). Ils sont évalués à la fois sur leur capacité à reproduire un climat statistique (modes de variabilité interne, etc.), mais aussi sur leur performance prédictive. Dans ce dernier cas, les observations sont injectées dans le modèle (on parle d'assimilation) pour construire un état initial des variables climatiques le plus proche possible de la réalité (on parle d'initialisation) à partir duquel l'intégration numérique, i.e. la prévision, peut commencer.

\section{Distinction entre prévision et projection climatique}

Dans les exercices successifs CMIP sur lesquels s'appuient les rapport du GIEC, les GCM ont été uniquement utilisés pour estimer la réponse du système climatique aux forçages externes (solaire, volcans, GES, etc.). Pour ce faire, un ensemble de simulations est traditionnellement réalisé ; une simulation déterministe, i.e. unique, n'a en effet pas de sens en science du climat de par la nature chaotique du système (sensibilité à d'infimes perturbations). L'approche ensembliste permet de prendre en compte cette propriété chaotique du système climatique : il s'agit de perturber très légèrement les conditions initiales et de réaliser ainsi plusieurs simulations (membres de l'ensemble), afin d'échantillonner le domaine des possibles et de quantifier une partie des incertitudes. Les perturbations sur les conditions initiales des simulations se justifient du fait que celles-ci ne sont pas parfaitement connues (incertitudes liées aux erreurs de mesure, à l'échantillonnage à la fois dans le temps et dans l'espace des observations, etc.) et que la représentation des processus dans le modèle n'est pas parfaite (incertitudes sur les lois de l'écoulement et des échanges d'énergie et de matière, etc.). 
Jusqu'à présent, les GCM n'ont pas été utilisés pour faire de la prévision climatique au-delà de l'échelle saisonnière. Même si les modèles reproduisent peu ou prou la fréquence et l'amplitude des variations de l'AMO ou de la PDO, aucune simulation n'a encore été conduite pour prévoir la chronologie des diverses phases du signal observé et en particulier sa modulation décennale. C'est pourquoi les termes "simulation historique » pour le $\mathrm{XX}^{\mathrm{e}}$ siècle et « projection climatique » pour le $\mathrm{XXI}^{\mathrm{e}}$ sont inscrits dans les rapports et non " prévision climatique ». Détail pour certains, cette distinction est centrale pour une interprétation correcte des conclusions fournies par la communauté des climatologues. Ainsi, reprocher au GIEC de ne pas avoir « prévu » l'infléchissement de la tendance au réchauffement des années 2000 par rapport à la décennie
1990 (les hypothèses privilégiées pour cette inflexion étant, en partie, le changement de phase de la PDO au début de la décennie qui tend à injecter davantage de chaleur dans l'océan profond, mais aussi la récurrence de « petites 》 éruptions volcaniques ; Solomon et al., 2011), ou encore la fin récente des grandes périodes de sécheresse au Sahel (celle-ci ayant en partie pour origine le changement de phase de l'AMO), relève d'une incompréhension totale de la nature même des projections climatiques et des simulations historiques sur lesquelles s'appuient les rapports successifs du GIEC depuis sa création, il y a maintenant 25 ans. "Prévoir " ce type d'inflexion n'était tout simplement pas dans le protocole de départ. Dans ce contexte, en quoi consiste la prévision décennale? Quelles en sont les particularités?

Figure 2 - Représentation schématique du protocole expérimental pour les projections/simulations historiques versus les prévisions décennales. Voir texte pour les détails.

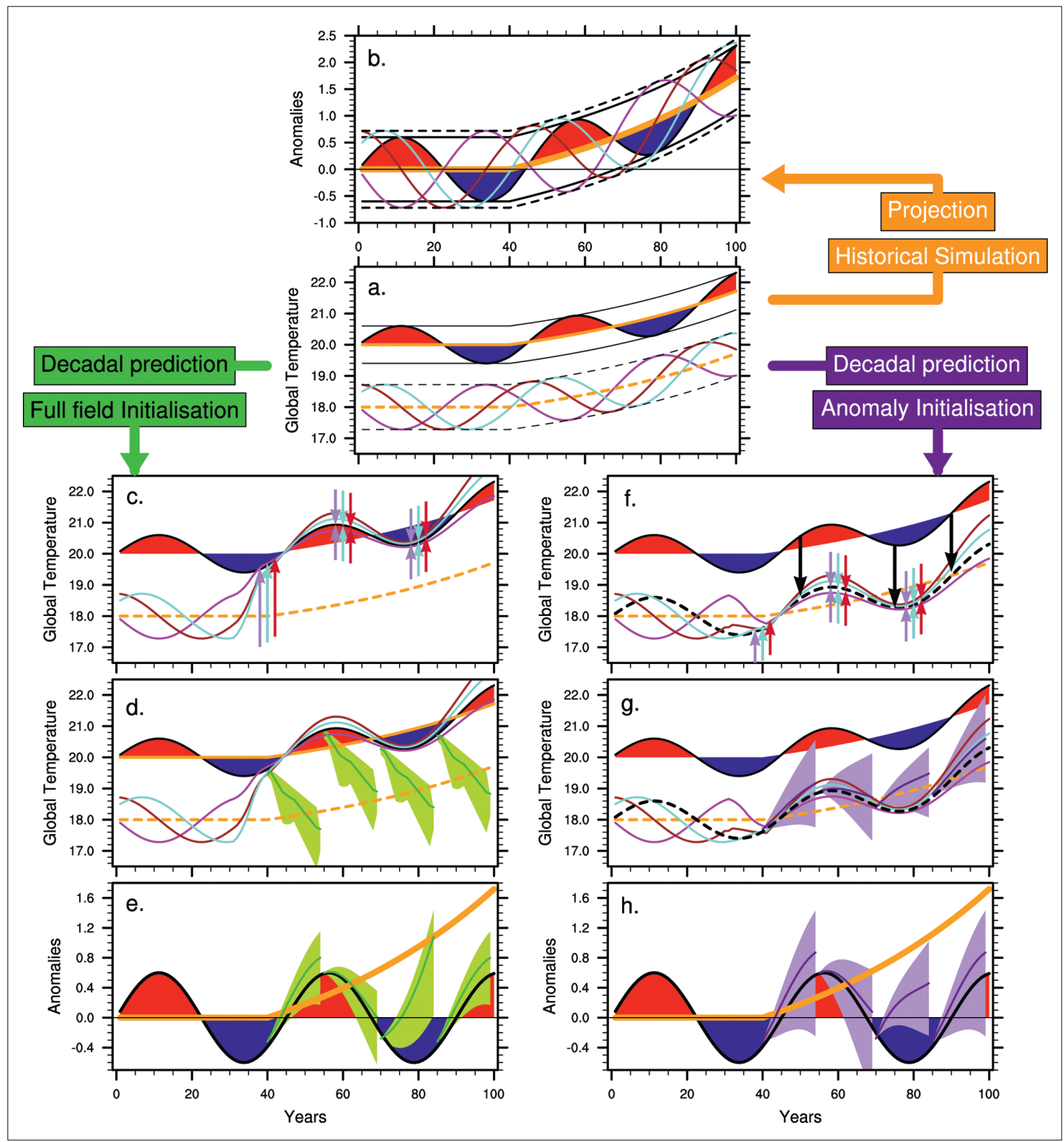

\section{Les diverses étapes de la prévision décennale}

Les modes de variabilité interne, tels l'AMO et la PDO, sont présents dans les GCM en tant que processus. Tout l'art de la prévision décennale consiste à prévoir leur chronologie afin de reproduire les modulations climatiques observées autour de la tendance due aux forçages externes. Un exercice de prévision se déroule ainsi en trois étapes :

1. L'initialisation du modèle. Il s'agit de produire l'instantané climatique correspondant aux observations à partir duquel le modèle est intégré pour prévoir. Dans le cas de l'Atlantique nord, par exemple (figure 1), on comprend que pour prévoir correctement la décennie 1985-1995, il est important de partir en 1985 d'une anomalie négative d'AMO cohérente avec les observations. L'initialisation de la composante océanique est essentielle, car c'est elle qui contient la mémoire du système climatique à l'échelle de temps considérée, de par sa forte capacité thermique et les constantes de temps des circulations. L'océan est donc l'acteur essentiel en prévision décennale avec, dans une moindre mesure, la glace de mer pour les régions polaires. Dans l'initialisation océanique réside ainsi la principale source de prévisibilité de première espèce. 2. L'intégration du modèle et la prise en compte des changements des conditions aux limites. Sur la période historique (1960-2010), l'évolution mesurée de la concentration des GES et les perturbations radiatives liées aux éruptions volcaniques, au cycle solaire et aux aérosols, sont imposées. Sur la période future (20102030), les scénarios d'émission de 
GES et de concentration d'aérosols, mais aussi les modulations potentielles de l'activité solaire (cycle à 11 ans) sont utilisés. Des éruptions volcaniques fictives, i.e. d'occurrence aléatoire, peuvent également être introduites afin de balayer tout le faisceau des possibles et ainsi estimer au mieux l'incertitude de la prévision. Cette étape correspond à la source de prévisibilité de deuxième espèce.

3. Le « débiaisage » (suppression du « biais » ou dérive du modèle). Il s'agit de corriger la prévision en enlevant $a$ posteriori les erreurs systématiques du modèle, afin d'extraire au mieux le signal décennal.

La figure 2 schématise ces trois étapes. Le signal observé (figure $2 \mathrm{a}$, courbe noire) correspond à la superposition d'une tendance (orange) et d'une modulation décennale (alternance bleu-rouge) de variance donnée (enveloppe trait noir). Le signal simulé en mode historique ou projection correspond à un ensemble de simulations matérialisées par les courbes de couleurs (ici trois membres, figure $2 a$ ) qui se superposent à une tendance (orange pointillé). Le domaine des possibles est représenté sur la figure par l'enveloppe (trait noir pointillé) permettant également de quantifier l'intensité de la variabilité interne du modèle (largeur de l'enveloppe, chaque projection développant sa propre chronologie de la variabilité interne) par rapport à la variabilité forcée ici commune à tous les membres de l'ensemble, c'est-à-dire la tendance.

Sur ce schéma, qui se trouve être assez représentatif du comportement de la plupart des GCM (chapitre $8 \mathrm{du} 4^{\mathrm{e}}$ rapport du GIEC ; Randall et al., 2007), nous voyons que le signal simulé en réponse aux forçages externes reproduit la tendance climatique (on dit que les modèles ont une « sensibilité climatique » correcte), mais souffre d'un biais moyen systématique, ici d'environ 2 degrés plus froid. Une manière de débiaiser le modèle est de travailler en anomalie par rapport à l'état moyen simulé et calculé sur une période donnée, en faisant alors l'hypothèse que les biais moyens n'interagissent ni avec la réponse du système climatique aux forçages externes, ni avec la variabilité interne. La figure $2 b$ schématise cette étape de débiaisage et correspond à un des graphes populaires communément repris dans les divers rapports du GIEC et pour la dissémination des résultats. Il représente l'estimation de la réponse du système climatique aux forçages externes obtenus à partir d'un ensemble de simulations historiques ou d'un ensemble de projections pour le futur.
L'étape additionnelle pour la prévision décennale repose sur l'initialisation ou phasage du modèle aux fluctuations observées, en particulier sa composante océanique (figure $2 \mathrm{cf}$ ). Plusieurs écoles coexistent sur le choix des variables à initialiser et sur les méthodes. L'approche dite " de surface » consiste à initialiser seulement les SST observées, ou leurs estimations, en mode couplé via un fort rappel (Keenlyside et al., 2008 ; Swingedouw et al., 2012). L'approche « tridimensionnelle » (Pohlmann et al., 2009 ; Kroger et al., 2012) consiste à initialiser la température et la salinité de l'océan par un rappel aux observations à la fois en surface et en profondeur. Les observations de subsurface sont cependant rares et leur couverture spatiale et temporelle peu homogène avant la fin des années 1970 (date de lancement des premiers satellites) et avant l'émergence des flotteurs Argo au début des années 2000. Dans le cas tridimensionnel, l'initialisation repose de ce fait sur les réanalyses océaniques produisant une estimation globale, cohérente et homogène de l'état océanique. Les progrès récents en la matière ont été majeurs grâce à la plus grande maturité des systèmes d'assimilation océanique, la qualité de la reconstruction des variables de forçage océaniques depuis 1960 via les réanalyses atmosphériques et/ou autres jeux de données et, bien sûr, grâce à l'amélioration des modèles eux-mêmes et la disponibilité d'observations récentes de très grande qualité ${ }^{(1)}$.

La technique la plus simple pour initialiser le modèle d'océan consiste à simplement interpoler le jeu d'observations sur la grille du modèle et se servir de cet état pour commencer la prévision ; on parle d'initialisation brut-force. Cette technique a l'avantage d'être relativement peu coûteuse en temps de calcul, mais elle est assez brutale, car l'état observé peut être relativement éloigné de l'état climatique moyen simulé par le modèle. Une autre technique consiste à contraindre le modèle à suivre les observations sur une fenêtre temporelle précédant la prévision afin de créer ses propres conditions initiales; on parle de nudging ou de « rappel » vers les observations. Cette étape est matérialisée par les flèches (figure $2 \mathrm{cf}$ ) et peut être effectuée soit à partir de l'observation brute (figure $2 \mathrm{c}$ ), le modèle étant ramené vers l'état moyen observé en sus de sa variabilité (panneaux de gauche, initialisation dite fullfield), soit sur l'anomalie de cette observation (figure $2 \mathrm{f}$ ) alors superposée à l'état moyen du modèle (panneaux de droite). Ces techniques de nudging présentent l'avantage d'aboutir à un état initial plus cohérent avec la dynamique du modèle ; on s'attend donc à un choc moins fort au moment de la prévision.

Un ensemble de prévisions de $\mathrm{N}$ membres est lancé sur 10 ans à partir des conditions initiales (enveloppe verte/ mauve et moyenne d'ensemble en trait plein, figure $2 \mathrm{dg}$ ). Dans le cas des initialisations full-field, le modèle retourne vers son état moyen (figure $2 \mathrm{~d}$ ) et cette dérive doit être systématiquement enlevée dans le signal prévu (figure 2e). La dérive est plus faible dans le cas de l'initialisation en anomalie (figure $2 \mathrm{~g}$ ) : elle correspond principalement au décalage spatial entre les anomalies observées et insérées dans les conditions initiales du modèle et les zones de variance propres au modèle.

La dernière étape consiste enfin à soustraire des prévisions débiaisées le signal lié aux forçages externes afin de quantifier la capacité du modèle à prévoir la chronologie des modes de variabilité interne (figure 2eh). Cette étape constitue la véritable valeur ajoutée de la prévision décennale par rapport aux simulations historiques et projections climatiques fournies jusqu'à maintenant ; elle quantifie l'impact de l'initialisation, c'est-à-dire l'importance du phasage des modes décennaux pour la prévision. Pour relever le défi de la prévisibilité sur les prochaines décennies, le PMRC a proposé pour la première fois, en plus des expériences classiques (simulations historiques et projections), une série d'expériences coordonnées dédiées à l'étude de la prévisibilité décennale. Cette initiative, sous la bannière CMIP5, constitue un guide pour l'élan international qui se met en place autour de la thématique émergente.

\section{L'exercice CMIP5}

Le cœur (ou CORE) du volet « décennal » de CMIP5 consiste à réaliser une série de hindcasts ou « prévisions rétrospectives » du climat des cinquante dernières années. Il s'agit de tenter de reproduire les fluctuations climatiques sur 10 années à partir de prévisions commencées tous les 0 et 5 ans de chaque décennie $(1960,1965,1970 \ldots)$ [figure 3 , cercle rose]. Le protocole propose que chaque lâcher soit constitué au minimum d'un ensemble de trois membres qui diffèrent par une petite perturbation de leurs conditions initiales selon l'approche ensembliste décrite plus

(1) Par abus de langage, dans le reste de l'article, les réanalyses océaniques seront inclues dans le terme observations. 


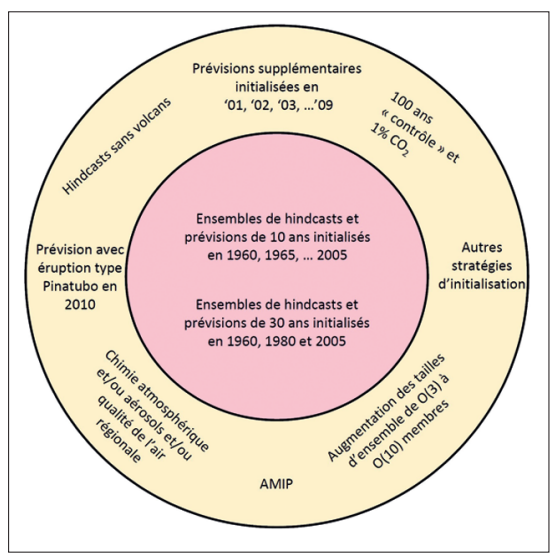

Figure 3 - Résumé schématique des expériences de prévision décennale de CMIP5, le CORE au centre en rose et les expériences additionnelles (en jaune) Extrait de Taylor et al. (2012).

haut. Ces prévisions rétrospectives ont pour but d'estimer le niveau de prévisibilité aux échelles décennales et de tester/valider/confronter le protocole d'initialisation mis en place par chacun des groupes sur les observations passées. Cette étape préliminaire est indispensable avant la véritable prévision sur 2010-2035. Des simulations additionnelles dites TIER1 (figure 3, cercle annulaire jaune) sont proposées afin de mieux comprendre les mécanismes physiques au cœur de la prévisibilité décennale (impact des volcans, etc.), mais aussi pour mieux estimer statistiquement cette prévisibilité (augmentation du nombre de membres, du nombre de dates de prévision, etc.). Au total, une quinzaine de groupes internationaux participe au volet décennal de CMIP5 et les sorties des 15 modèles sont mises à disposition sans restriction sur tous les sites de diffusion de données climatiques.

Si l'exercice est contrôlé pour le type d'expériences à mener, il est en revanche totalement libre sur les méthodologies à employer, en particulier pour l'initialisation océanique. Il en ressort que les 15 groupes ont choisi, selon leurs propres contraintes et expertises, un type d'initialisation parmi celles disponibles, à savoir brut-force ou nudging, SST ou contenu thermique/halin tridimensionnel, anomalie ou full-field, initialisation atmosphérique ou non, initialisation de la glace de mer ou non, etc. Cette diversité doit être présente à l'esprit lorsque les analyses multimodèles sont produites et interprétées. Cette diversité révèle tout le caractère « exploratoire » de ce premier exercice international en matière de prévision décennale. Celui-ci doit être considéré comme une première tentative, loin de la maturité des simulations historiques et des projections qui alimentent les rapports du GIEC depuis 25 ans.

\section{Premiers résultats sur la prévisibilité décennale}

D'un point de vue théorique, les premières études ont commencé à fleurir dans la littérature à la fin des années 2000. On y trouve des papiers généraux sur la pertinence et la faisabilité de l'exercice lui-même (Hawkins et Sutton, 2009 ; Hurrell et al., 2009) et sur les premières tentatives de prévision décennale. La faisabilité, ou la mise en évidence d'une forme de prévisibilité, a souvent été suggérée par l'approche dite "modèle parfait 》. Il s'agit d'estimer si les modèles sont capables de prévoir leur propre variabilité décennale, avant de savoir s'ils le sont pour les variations observées. Sur ce point, le projet européen FP5PREDICATE fut pionnier et suggère un potentiel prédictif fort de la circulation océanique de grande échelle dans l'océan Atlantique (AMOC) [Collins et al., 2006]. Ces conclusions sont confirmées dans des communications plus récentes utilisant les dernières versions des modèles du NCAR et du GFDL (Hurrell et al., 2009) développés aux États-Unis ou du MetOffice britannique (Dunstone et Smith, 2010). Les premières tentatives de prévision réelles démontrent, sur de nombreux paramètres physiques, que l'assimilation des données océaniques pour l'initialisation des prévisions est une véritable valeur ajoutée (cf. le projet européen FP6-ENSEMBLES, Zhang et al., 2010) sur des régions particulières. Les études pionnières sont celles de Smith et al. (2007), Keenlyside et al. (2008), Pohlmann et al. (2009) ou encore van Oldenborgh et al. (2012), parmi d'autres.

Figure 4 - Coefficient de corrélation calculé sur la période 1960-2010 pour la température à 2 mètres, aux échéances 2-5 ans dans les prévisions décennales du CNRM-CERFACS (en haut) et de I'IPSL (en bas), après avoir soustrait les tendances locales par régression linéaire sur la concentration du $\mathrm{CO}_{2}$, à la fois dans le modèle et dans le jeu d'observation HadCRU3v. Les valeurs significatives au seuil $95 \%$ selon un r-test sont représentées par un point et les régions où les observations sont insuffisantes pour le calcul sont grisées.
Dans CMIP5, il apparaît qu'une grande partie de la prévisibilité décennale sur la température de surface provient des forçages externes (prévisibilité de la deuxième espèce). Globalement, le rôle des GES, des aérosols volcaniques et anthropiques est dominant, assurant une prévisibilité forte pour les quantités très intégratrices (SST, contenu de chaleur océanique, etc.). Régionalement, l'initialisation de l'océan semble en revanche être essentielle pour reproduire et prévoir les fluctuations observées, en particulier celles de l'AMO. Un score de prévision classique consiste à mesurer la corrélation des prévisions rétrospectives avec les observations correspondantes. La figure 4 fournit ce score pour la température à 2 mètres, une fois la réponse forcée retirée ; elle confirme une prévisibilité forte et significative due à l'initialisation océanique pour les deux modèles sur l'Atlantique, en particulier dans les régions subtropicales et sur le bord est du bassin à l'échéance 2-5 ans. Dans le modèle du CNRM-CERFACS, les scores sont aussi élevés dans le gyre subpolaire grâce à l'initialisation tridimensionnelle océanique, alors que l'IPSL a adopté l'approche de surface (voir plus haut). Les scores sont aussi positifs dans l'océan Indien et l'ouest du bassin pacifique tropical, mais la prévisibilité est quasi inexistante ailleurs, en particulier sur le Pacifique (confirmé par des scores très faibles pour la PDO ; Kim et al., 2012).

La capacité prédictive déborde sur les continents adjacents aux zones océaniques aux scores élevés. On trouve

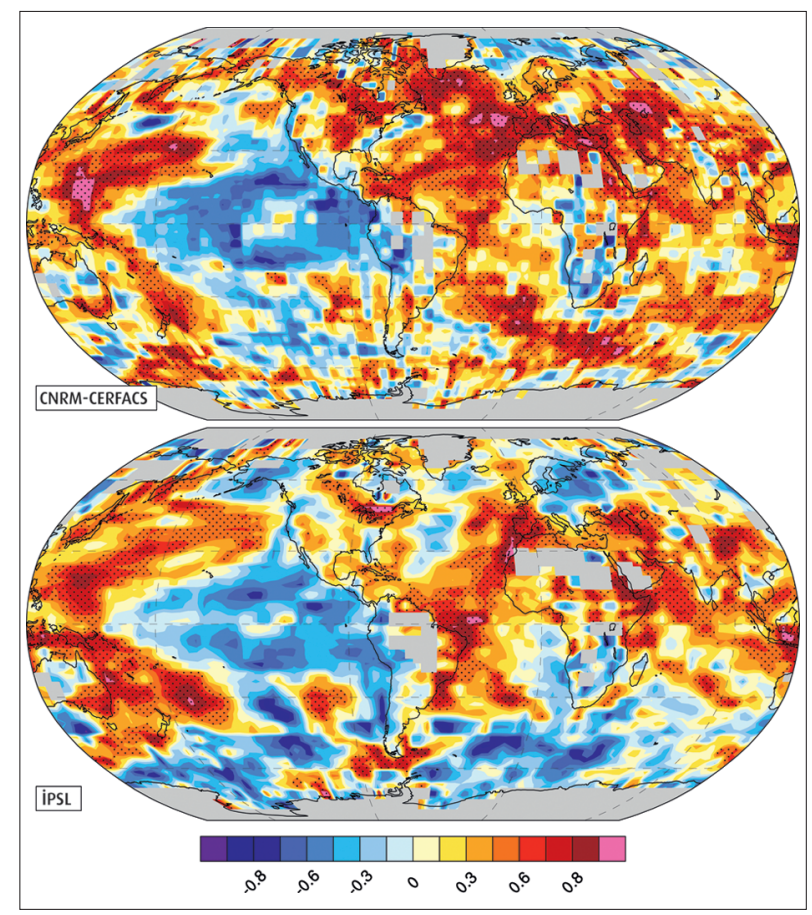


ainsi dans les deux modèles des corrélations significatives entre prévision et observations aux échéances 2-5 ans sur l'Europe de l'Ouest, sur tout le pourtour méditerranéen jusqu'aux plaines de l'Asie centrale, la corne de l'Afrique. De manière générale, les scores de prévision sont cependant beaucoup plus faibles sur les continents que sur les océans. En termes de précipitations, les scores sont pratiquement nuls sur l'ensemble de la planète dans les deux modèles.

Insistons sur le caractère très préliminaire de ces résultats. Des réflexions sur les statistiques et méthodes sont en cours pour fournir des techniques plus adaptées que celles d'aujourd'hui pour déconvoluer le signal forcé du signal interne, pour tenir compte de la petitesse du nombre d'échantillons dans l'estimation des scores de prévision et leur robustesse physique et statistique, pour s'affranchir des biais et dérives des modèles, etc. Nous sommes en prévision décennale au niveau de connaissance et de capacité technique de la prévision saisonnière numérique par des modèles couplés océan-atmosphère lors de ses réels débuts dans les années 1990. Les progrès ont été considérables depuis.

\section{Discussion}

La prévision décennale constitue un véritable défi pour la communauté des climatologues (communauté amont en tant que fournisseurs d'information climatique), mais aussi pour la communauté des impacts/décideurs/acteurs socio-économiques/citoyens (communauté aval en tant que utilisateur/client de cette information). Ces deux communautés doivent construire une grille de lecture commune, un point de rencontre entre une demande croissante et légitime de produits climatiques et une connaissance scientifique du système et des méthodes encore embryonnaires aux échelles décennales. Il est de la responsabilité de la communauté climatique de ne pas survendre les performances et les connaissances en matière de prévision décennale. Il est de la responsabilité de la communauté des utilisateurs de ne pas surinterpréter les résultats et de s'approprier les problématiques et limites inhérentes de l'exercice afin de ne pas demander l'impossible aux sciences du climat. Les Anglo-Saxons parlent souvent de développer une véritable climate literacy que 1'on pourrait traduire par
« alphabétisation en sciences du climat ». Cet article s'inscrit dans ce cadre avec le choix de porter l'attention sur la problématique, les méthodes et les limites de la prévision décennale, ou encore sur l'estimation de la prévisibilité, véritable prérequis, plutôt que de décrire les résultats de la prévision des prochaines décennies. D'un point de vue scientifique, les expériences de « prévisions rétrospectives », celles couvrant les périodes pour lesquelles des observations sont disponibles, concentrent tout autant d'intérêt que celles se focalisant sur la période future. Elles permettent en particulier de mieux comprendre les biais des modèles et leurs échelles de temps et d'espace caractéristiques.

Le volet décennal de l'exercice CMIP5 est une première et doit être considéré comme tel, à savoir une tentative, un exercice de recherche fondamentale, un véritable tremplin vers la connaissance des mécanismes physiques qui gouvernent le climat aux échelles décennales. Il s'agit dans cet exercice de prévoir la tendance climatique en réponse aux forçages anthropiques et sa modulation par la variabilité interne (principalement océanique). La complexité de l'exercice est grande, car l'échelle décennale semble concentrer toutes les limitations des études climatiques, à savoir : la couverture observationnelle limitée dans le temps et dans l'espace, ce qui rend l'estimation des fluctuations décennales et la validation même de leur prévision difficiles, l'interaction entre les biais et les dérives des modèles et la variabilité/ prévisibilité à ces échelles de temps, la coexistence de différentes sources de variabilité/prévisibilité qui interagissent.

Les premiers résultats suggèrent que la prévisibilité associée aux forçages externes est forte et planétaire, alors que celle des modes internes dépend du bassin océanique et a une signature régionale. Ainsi, l'Atlantique nord (ou plus précisément l'AMO) est plus prévisible que le Pacifique (en particulier la PDO), avec des scores de prévision significatifs jusqu'à 7-8 ans pour le premier (van Oldenborgh et al., 2012) et seulement 2-3 ans pour le second (Kim et al., 2012). Les variables continentales (température, précipitation) semblent peu prévisibles en général, suggérant la nécessité de passer par des approches statistiques et de descente d'échelles construites à partir des observations océan-continent pour en déduire le signal décennal prévu.
Les résultats de CMIP5 ouvrent de nombreux chantiers, posent des questions essentielles et insistent sur les priorités de recherche à venir. Il en ressort que :

- le travail sur la compréhension des processus, des mécanismes et rétroactions fondamentales associés aux échelles décennales dans un contexte de changement climatique, doit être au cœur des futurs axes de recherche ;

- le travail sur les méthodes est essentiel pour améliorer l'initialisation de l'océan et de la glace de mer, la prise en compte du forçage épisodique lié aux éruptions volcaniques, la prise en compte du caractère régional des aérosols d'origine anthropique qui jouerait sur la variabilité interne et son estimation, en particulier sur l'AMO (Booth et al., 2012 ; Terray, 2012), etc. L'hypothèse de séparabilité entre la variabilité forcée et la variabilité interne doit être clairement documentée ;

- le travail sur les modèles est primordial afin de mieux simuler les modes de variabilité interannuels à décennaux, minimiser les biais et les dérives qui polluent le signal à prévoir. Il s'accompagne d'un travail sur l'estimation même de la prévisibilité, étant donnés ces biais et dérives ;

- le travail sur les réanalyses de tous les compartiments du système climatique est essentiel pour les étapes de validation et, bien évidemment, pour l'initialisation. Les initiatives visant à étendre les réanalyses dans le passé doivent être clairement soutenues ;

- la mise en adéquation des moyens avec les contraintes inhérentes à la physique de l'échelle décennale, à la fois en termes de ressources de calcul/stockage et de disponibilité des observations, est indispensable. Ainsi, pour débiaiser correctement les modèles et pour bien séparer et prévoir les signaux forcé et interne, il est obligatoire de conduire un très grand nombre d'expériences et d'aller bien au-delà du CORE et même du TIER1 de CMIP5. Les groupes français sont fortement pénalisés dans la disponibilité des ressources numériques nationales en la matière. Concernant les observations, il est vital au minimum de consolider le système observationnel actuel, car des ruptures dans les séries dues à des défaillances instrumentales seraient désastreuses et excluraient tout progrès en matière de prévision décennale. Bien entendu, la mise à disposition de nouveaux jeux de données (salinité mesurée par satellite - missions AQUARIUS et SMOS -, dynamique des calottes, déploiement de nouveaux profileurs océaniques, etc.) ouvrira de nouvelles perspectives et opportunités de progrès ; 
- fournir à la communauté des impacts un cadre et une expertise sur l'utilisation des données de prévision décennale (qui ne peuvent en aucun cas être utilisées sans traitement préalable, en particulier pour les applications régionales) est essentiel. La présence de dérive en mode prévision, alors que celle-ci n'existait pas dans les projections utilisées jusqu'alors, complexifie grandement le problème, en particulier pour les valeurs journalières et les études sur les événements extrêmes (vagues de chaleur, lames d'eau, etc.).

Comme déjà mentionné, tous ces efforts et initiatives constituent un véritable défi pour la communauté des climatologues au sens large, mais ils sont un prérequis avant toute prévision décennale opérationnelle, même si celle-ci doit être anticipée dès aujourd'hui. Le projet européen FP7-SPECS (Seasonal-to-decadal climate Prediction for the improvement of European Climate Services) a été lancé en novembre 2012 - dans lequel sont impliqués le CERFACS et l'IPSL sur le thème décennal - devrait permettre de concentrer les efforts européens pour avancer un pas de plus dans cette voie.

\section{Remerciements}

Les travaux et analyses présentés ont été réalisés dans le cadre du projet EPIDOM (Étude de la prévisibilité interannuelle à décennale à partir des observations et des modèles) financé par le programme GICC (Gestion des impacts du changement climatique) sous la tutelle du MEDDTL (ministère de l'Écologie, du Développement durable, des Transports et du Logement), ainsi que du projet SPECS, financé par la Commission européenne à travers le septième programmecadre pour la recherche (thème 6 : Environnement), accord 303837, en cours de novembre 2012 à janvier 2017. Les auteurs souhaitent remercier pour leur aide tous les acteurs de la thématique décennale à la fois au CERFACS et à l'IPSL, ainsi que l'ensemble des équipes impliquées dans le développement des deux modèles de climat français.

\section{Bibliographie}

Booth B. B. B., N. J. Dunstone, P. R. Halloran, T. Andrews et N. Bellouin, 2012 : Aerosols implicated as a prime driver of twentieth-century North Atlantic climate variability. Nature, 484, 228-232, doi:10.1038/nature10946.

- Collins M., N. Botzet, A. F. Carril, H. Drange, A. Jouzeau, M. Latif, S. Masima, O. H. Otteraa, H. Pohlmann, A. Sorteberg, R. Sutton et L. Terray, 2006 : : Interannual to Decadal Climate Predictability in the North Atlantic: A Multimodel-Ensemble Study. J. Climate, 19, 1195-1203.

: Déqué M., 2003 : La prévision numérique à l'échelle saisonnière : que sait-on faire et que peut-on espérer ? La Météorologie, 8 série, 41, 20-29.

: Dunstone N. J. et D. M. Smith, 2010 : Impact of atmosphere and sub-surface ocean data on decadal climate prediction. Geophys. Res. Lett., 37, L02709, doi:10.1029/2009GL041609.

: Hawkins E. et R. Sutton, 2009 : The potential to narrow uncertainty in regional climate predictions. Bull. Amer. Meteor. Soc., 90, 1095-1107, doi:10.1175/2009BAMS2607.1.

- Hurrell J., G. A. Meehl, D. Bader, T. L. Delworth, B. Kirtman et B. Wielicki, 2009 : A unified modeling approach to climate system prediction. Bull. Amer. Meteor. Soc., 90, 1819-1832, doi:10.1175/ 2009BAMS2752.1.

Keenlyside N., M. Latif, J. Junclaus, L. Kornblueh et E. Roeckner, 2008 : Advancing decadal climate scale prediction in the North Atlantic. Nature, 453, 84-88.

Kerr R. A., 2000: A North Atlantic climate pacemaker for the centuries. Science, 288, 1984-2000.

: Kim H-M., P. Webster et J. A. Curry, 2012 : Evaluation of short term climate prediction in multi-model CMIP5 decadal hindcasts. Geophys. Res. Lett., 39, L010701, doi:10.1029/2012GL051644

Knight J. R., R. J. Allan, C. K. Folland, M. Vellinga et M. E. Mann, 2005 : A signature of persistent natural thermohaline circulation cycles in observed climate. Geophys. Res. Lett., 32, L20708, doi:10.1029/2005GL024233.

Kröger J., W. Müller et J.-S. von Storch, 2012 : Impact of different ocean reanalyses on decadal climate prediction. Clim. Dyn., 39, 795-810, doi:10.1007/s00382-012: $1310-7$.

- Lorenz E. N., 1975 : Climatic predictability. Dans The Physical Basis of Climate and Climate Modelling, B. Bolin et al., Eds., GARP Publication Series, 16, World Meteorological Organization, 132-136.

: Mantua N. J., S. R. Hare, Y. Zhang, J. M. Wallace et R. C. Francis, 1997 : A Pacific decadal climate oscillation with impacts on salmon. Bull. Amer. Meteor. Soc, 78 , 1069-1079

: Pohlmann H., J. H. Jungclaus, A. Köhl, D. Stammer et J. Marotzke, 2009 : Initializing decadal climate predictions with the GECCO oceanic synthesis: Effects on the North Atlantic. J. Climate, 22, 3926-3938, doi:10.1175/ 2009JCLI2535.1.

Randall D. A. et al., 2007 : Climate models and their evaluation. Dans Climate Change 2007: the physical Science Basis. Cambridge University Press, Cambridge, United Kingdom and New York, NY, USA.

Smith D. M., S. Cusack, A. W. Colman, C. K. Folland, G. R. Harris et J. M. Murphy, 2007 : Improved surface temperature prediction for the coming decade from a global climate model. Science, 317, 796-799.

Solomon S., J. S. Daniel, R. R. Neely, J-P. Vernier, E. G. Dutton et L. W. Thomason, 2011 : The persistently variable background stratospheric aerosol layer and global change. Science, 333, 866-870, doi:10.1126/science.1206027.

Sutton T. W. et B. Dong, 2012 : Atlantic Ocean influence on a shift in European climate in the 1990s. Nature Geosci., 5, 788-792, doi:10.1038/ngeo1595.

Swingedouw D., J. Mignot, S. Labetoulle, E. Guilyardi et G. Madec, 2013 : Initialisation and predictability of the AMOC over the last 50 years in a climate model. Clim. Dyn., 40, 2381-2399, doi:10.1007/s00382-012-1516-8.

Taylor K. E., R. J. Stouffer et G. A. Meehl, 2012 : An overview of cmip5 and the experiment design. Bull. Amer. Meteor. Soc., 93, 485-498, doi:10.1175/BAMS-D-11-00094.1.

Terray L., 2012 : Evidence for multiple drivers of North Atlantic multi-decadal climate variability. Geophys. Res. Lett., 39,L19712, doi:10.1029/2012GL053046.

Trenberth K. E. et D. J. Shea, 2006 : Atlantic hurricanes and natural variability in 2005. Geophys. Res. Lett., 33, L12704, doi:10.1029/2006GL026894.

- van Oldenborgh G. J., F. J. Doblas-Reyes, B. Woulters et W. Hazeleger, 2012 : Skill in the trend and internal variability in a multi-model decadal prediction ensemble. : Clim. Dyn., 38, 7, 1263-1290, doi:10.1007/s00382-012-1313-4.

: Zhang R. et T. L. Delworth, 2006 : Impact of Atlantic multidecadal oscillations on India/Sahel rainfall and Atlantic hurricanes. Geophys. Res. Lett., 33, L17712, doi:10.1029/2006GL026267.

Zhang S., A. Rosati et T. L. Delworth, 2010 : The adequacy of observing systems in monitoring AMOC and North Atlantic climate. J. Climate, 23, 5311-5324, doi:10.1175/2010JCLI3677.1. 